\title{
DAS VERKEHRSNETZ DER SCHWEIZ UND DIE NATIONALSTRASSEN
}

\author{
ERNST WiNKLER
}

Mit dem Schlußbericht der Kommission des eidg. Departements des Innern für die Planung des Hauptstraßennetzes «Die Planung des schweizerischen Nationalstraßennetzes»* wurde die erste, die grundlegende Etappe eines großen Werkes abgeschlossen. Dieses Werk wird, einmal gebaut, so weiträumige und nachhaltige Einflüsse auf die Kulturlandschaft der Schweiz ausüben, daß auch die Geographie, die Landschaftsforschung an ihm nicht vorüber gehen kann. Es soll daher versucht werden, das bisher Geleistete knapp zu würdigen.

Ein solcher Versuch hat davon auszugehen, daß der Straßenbau in der Schweiz: vor allem die Befugnis, das Netz der öffentlichen Straßen zu bestimmen, die Straßen zu bauen, zu unterhalten und dem Verkehr dienstbar zu machen, nach dem geltenden Bundesrecht grundsätzlich in der Hoheit der Kantone liegt. Die Anlage von nationalen Straßen bedeutet also ein Novum in der Verkehrsgeschichte unseres Landes. Immerhin bestanden bereits seit Inkrafttreten der Bundesverfassung von 1848 eine Reihe von Einschränkungen der Kantonshoheit, die mit zunehmendem Verkehr vermehrt wurden.

Hatte der Bund mit Art. 37 der Bundesverfassung das Recht der Oberaufsicht über die Straßen und Brücken, an deren Erhaltung die Eidgenossenschaft ein besonderes (militärisches und politisches) Interesse besitzt, so wurde ihm 1922 noch die Befugnis übertragen, den Motorfahrzeug- und Fahrradverkehr zu ordnen und die Öffnung bestimmter Straßen für den allgemeinen Durchgangsverkehr zu erwirken. Ihm stand auch das Recht zu, im Interesse des Landes eigene öffentliche Werke zu errichten, oder deren Bau zu unterstützen, was beispielsweise durch Beiträge an die Erstellung der Brünigstraße (1856), Grimsel- und Klausenpa B (1891) oder Gandriastraße (1930) geschah. Die ungemeine Verkehrsintensivierung der letzten Jahrzehnte und die Tatsache, daß der Bund durch Erhebung eines Benzinzolls einen beträchtlichen Teil der fiskalischen Leistungsfähigkeit des Motorfahrzeugs abschöpfte, während er den Kantonen die steigenden Straßenbaulasten überließ, drängten jedoch zu grundlegend neuen finanzpolitischen aber auch technischen Lösungen.

Ihre Verwirklichung begann 1928 mit der Zuweisung der Hälfte des Zuschlagszolls auf Treibstoffe, des sog. «Benzinzollviertels», an die Kantone mit der Auflage, ihn für die Verbesserung und den Ausbau der dem Autoverkehr dienenden öffentlichen Straßen zu verwenden. Doch setzte erst 1935 mit dem Bundesbeschluß über den Ausbau der Straßen und des Straßennetzes im Alpengebiet eine eigentliche Straßenbaupolitik ein, die 1953 und 1955 zur Festlegung eines Hauptstraßennetzes mit Ausbaurichtlinien und Normalien führte. Schon in den hierbei angestellten Erwägungen der Baubehörden wurde auf die Notwendigkeit einer allgemeinen Straßenplanung hingewiesen, die der eigentlichen Projektierung voranzugehen habe. Auf Grund einer Eingabe der Baudirektorenkonferenz und der Studienkommission für den schweizerischen Hauptstraßenbau (26. Mai 1954) und nicht zuletzt zahlreicher Wünsche aus dem ganzen Volke setzte das Eidg. Departement des Innern am 15. September 1954 eine Kommission ein, die alle mit der Staßenplanung zusammenhängenden technischen, finanziellen, wirtschaftlichen, verkehrspolitischen und rechtlichen Fragen abklären sollte.

Im besonderen lauteten die Fragen: Auf welchen Verbindungen des vom Bundesrat bezeichneten Hauptstraßennetzes sind Autobahnen oder autobahnähnliche Anlagen notwendig? Wie sind diese Straßenzüge im Gelände anzulegen und wie soll ihre Verbindung mit dem übrigen Straßennetz, namentlich im Gebiete der Städte, vorgesehen werden? Inwieweit bedürfen die schon früher festgelegten Normalien für diese Neuanlagen noch einer Ergänzung oder Abänderung? Nach welchem örtlichen und zeitlichen Bauprogramm ist der Ausbau des Hauptstraßennetzes durchzuführen? Wo kommen in der Schweiz längere Straßentunnel zur Ermöglichung des wintersicheren Verkehrs durch die Alpen in Frage? Inwieweit bedarf die bestehende Gesetzgebung zur Verwirklichung der vorgenannten Neuanlagen und zur Erhaltung des Charakters gut ausgebauter Straßen als Durchgangsstraßen einer Abänderung oder Ergänzung? Wie sind Autobahnen und andere größere, im Rahmen des Bundesprogrammes auszuführende Straßenbauten zu finanzieren? Bedarf das schweizerische Straßenwesen zur Lösung dieser Aufgabe einer grundsätzlichen Neuordnung und welcher? Dabei wurde der Kommission

* Bern 1959. Eidg. Drucksachen- und Materialzentrale. 
freigestellt, sich noch zu andern im Zusammenhang auftauchenden Problemen Stellung zu beziehen. Dagegen hatte sie die Beziehungen zu andern Verkehrsträgern ausser Acht zu lassen.

Die Planungskommission konstituierte sich am 23. November 1954 und bildete mehrere Ausschüsse (allgemeine Verkehrspolitik, Planung, Finanz-, Rechts- und Organisationsfragen, Verkehrsgrundlagen für Tal- und Alpenstraßen, technische Planung der Talstraßen, Fragen der Alpentunnels, Aufstellung des Bauprogramms, Regional- und Städtegruppen). Sie nahm bei der Ernennung der Kommissionsmitglieder Bedacht auf gebührende Berücksichtigung aller an der Planung interessierten Kreise.

Geographie und Landesplanung erhielten in diesem Zusammenhang die Genugtuung, daß einer ihrer namhaftesten Vertreter, Prof. Dr. H. Gutersohn, Ordinarius für Geographie an der Eidg. Technischen Hochschule und Präsident der Schweizerischen Vereinigung für Landesplanung, als Leiter eines der wichtigsten Ausschüsse (für Alpenstraßen) berufen wurde. Auch sonst kam, wie der eingangs genannte Bericht belegt, die Landschaftsforschung zur Geltung, indem an den Ausgangspunkt der Planung die verkehrsgeographischen Gegebenheiten gestellt und insbesondere wegweisende Arbeiten eines weitern Schweizer Geographen über die zentralen Orte, H. Carols, eingebaut wurden. Das Präsidium der Kommission lag bei Ständeund Regierungsrat G. WENK und nach seinem vorzeitigen Ableben bei National- und Regierungsrat S. BRAWAND. Der Oberbauinspektor Dr. R. RUCKLI, dessen Sekretariat die Geschäfte führte, «war die eigentliche treibende Kraft und die Seele der Arbeiten, denen er auch die wesentlichen wissenschaftlichen Grundlagen zu schaffen verstand» (Bundesrat ETTER).

Anfänglich wurde geglaubt, daß die Planung innert eines Jahres abgeschlossen werden könne. Doch zeigte sich bald, $\mathrm{da} B$ sie wesentlich mehr Zeit benötigte, da in weiten Gebieten Neuland betreten und zahlreiche Einzelfragen abgeklärt werden mußten. Dennoch ist erstaunlich, da $\beta$ im wesentlichen im Sommer 1957 das Studium beendet war, so daß an die Ausarbeitung des Schlußberichtes gegangen werden konnte. Er wurde am 20. Mai 1959 dem damaligen Chef des Eidg. Departementes des Innern, Dr. Ph. ETter, eingereicht. Er besteht aus 6 Bänden, von denen der vierte über städtische Expreßstraßen als Verbindungsstrecken der Autobahnen noch aussteht. Den Kern des großen Gutachtens macht der in den Bänden 2 und 3 niedergelegte Teil über die eigentliche «Planung des Autobahnnetzes» aus, den allgemeine (Grundlagen-) Betrachtungen, Untersuchungen über die «Straßenverbindungen über die Alpen», über die «Festlegung des Nationalstraßennetzes». Bauprogramm, Kosten, Finanzierung, volkswirtschaftliche Auswirkungen der Autobahnen und ein Band Gesetzgebung (Bd. 6) einrahmen. Die benützten Pläne (1:25 000 und 1:5000) konnten dem Bericht ihres Umfanges wegen begreiflicherweise nicht beigegeben werden; sie sind durch Übersichtskarten 1:300 000 ersetzt, die das Verständnis der Planung jedoch durchaus ermöglichen.

«Jeder geographische Überblick unseres Landes (und auch jede Verkehrsüberlegung!) muß vom Relief ausgehen, denn weil die orographischen Verhältnisse sehr verschiedenartig und einflußreich sind, wirken sie sich in allen Belangen der Kulturlandschaft in starkem Maße aus». Mit diesem Einleitungssatz des Gesamtberichtes ist ausgedrückt, das zwei landschaftliche Phänomene vor allem: Bodengestalt und Kulturlandschaft auch von den Straßenfachleuten als grundlegende Faktoren ihrer Gestaltungsabsichten anerkannt wurden. Wenn sie in der Folge wirtschaftlichen Erwägungen entscheidendes Gewicht bei der Beurteilung der zu planenden Verkehrsstränge beimaßen und hierbei dem Mittelland als der bevorzugten Region den Hauptteil zuerkannten, war dies lediglich Ausdruck jener grundlegenden Tatsache der einmaligen Reliefstruktur und ihrer kulturlandschaftlichen Ausprägung. Landbau wie Industrie, Tourismus wie Volksdichte und zentrale Orte wie der Verkehr selbst (welch letztere beiden im Bericht merkwürdigerweise nicht zur «Geographie» des Landes gerechnet werden) sind in diesem komplexen Gefüge ja nur Elemente und (Einzel-) Faktoren, auf die eine Straßenplanung indes entscheidende koordinative Rücksicht zu nehmen hatte. Letzten Endes entschied aber der Verkehrsstrom, d. h. seine raumzeitliche Differenzierung bzw. Massierung über die Fixierung der Autobahnen und ihre regionale Verteilung.

Das schließlich beschlossene Nationalstraßennetz, das bis 1980 errichtet werden soll, erweist sich denn auch als ausgesprochenes Spiegelbild der «Verkehrsbelastung» (s. Karten) der bestehenden Straßen, wenn auch im Einzelnen Abweichungen von letztern vorgenommen wurden. Selbst die Bevorzugung der großen Städte als Schwer- und damit Verbindungspunkte (Zürich, Basel, Bern, Lausanne, Genf) der eigentlichen Autobahnen oder Nationalstraßen erster Klasse, die diesen «Spiegel» etwas zu ver- 

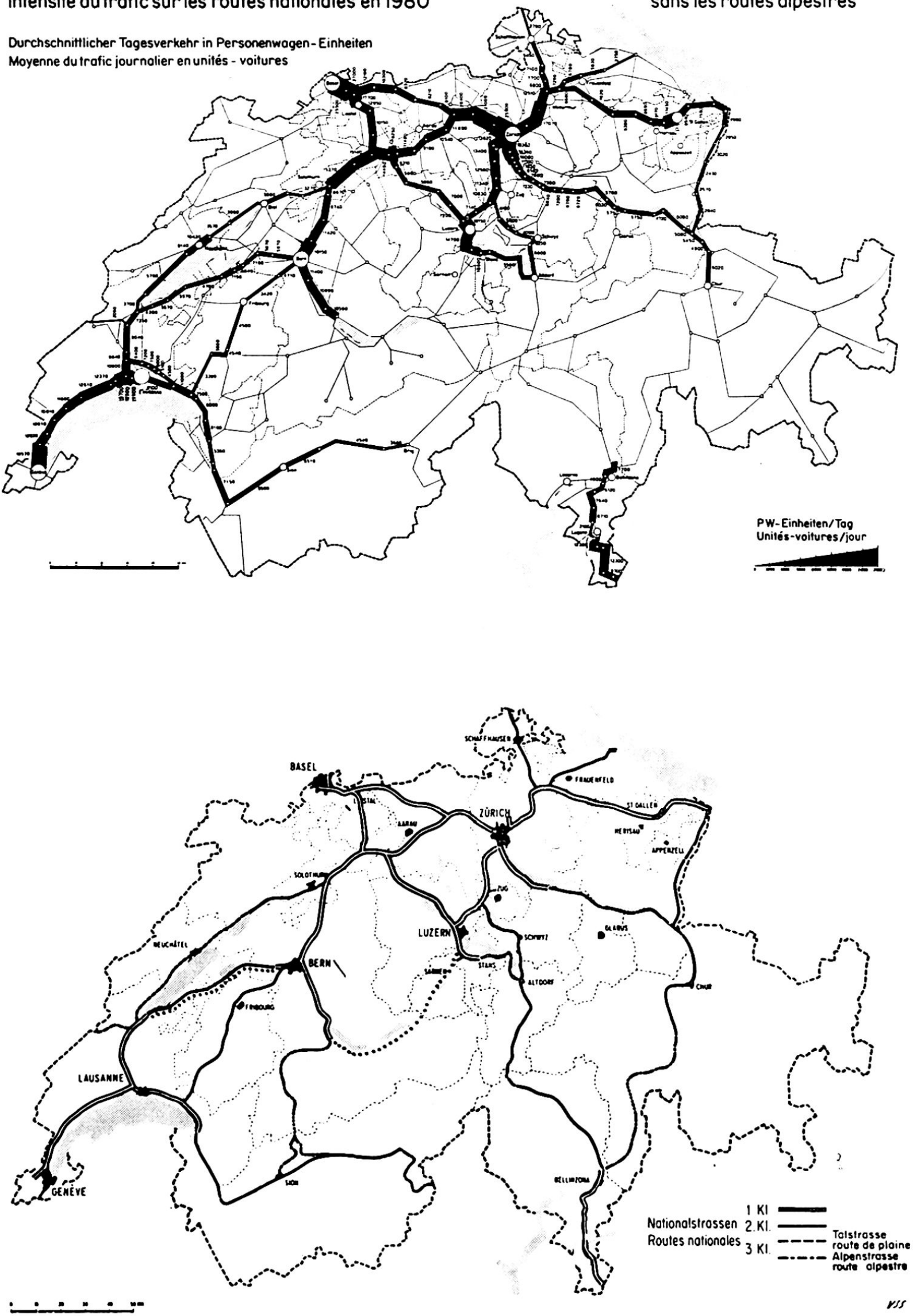

Das Nationalstraßennetz nach dem Vorschlag der Planungskommission, Zustand 1980. Punktierte Linien: nachträgliche Ergänzungen. Mit freundlicher Erlaubnis der Redaktion der Zeitschrift «Straße und Verkehr» 46, 1960 Nr. 1, entnommen. 
zerren scheint, bedeutet im Grunde sogar eine Betonung der genannten Tatsache. Sie kommt im - übrigens durchaus historischen - mehrfachen Kreuz von Westost- und Nordsüd-Linien und von Stadtverbindungsstraßen zum Ausdruck, welch letztere ja vor allem Ziel eines Autobahnnetzes, sogar in internationaler Sicht, gewesen sind. Wie viele Einzelüberlegungen indes notwendig waren, um $\mathrm{zu}$ diesem relativ einfachen Schluß zu gelangen, vermag nur ein eingehendes Studium des Berichtes selbst schlüssig verständlich machen. Wenn auch dessen Beurteilung im einzelnen dadurch erschwert erscheint, daß seine Beweisführung allzu kursorisch publiziert wurde (wie zum Beispiel die Erörterungen über Beziehungsdichte, Beziehungsgefälle, Erschließungs- und Anschlußwerte oder vor allem diejenigen über die Bedeutung der zentralen Orte. erkennen lassen, die mangels hinreichender zahlenmäßiger Untermauerung mehr Fragen stellen als lösen), überzeugt mindestens die Verkehrsanalyse und -prognose als ein Werk zuverläßiger Ingenieurarbeit.

Vielfach mußte hierbei, nicht zuletzt in terminologischen Fragen, Neuland betreten werden, wie bereits der Grundbegriff «Nationalstraßen» andeutet. Dieser wurde geprägt, um das von der Planungskommission zum Ausbau vorgeschlagene Straßennetz von den übrigen Verkehrswegen zu unterscheiden und wohl auch, um größere Freiheit gegenüber technischen Gestaltung, insbesondere im Blick auf den Terminus «Autobahn» zu haben. Aus verschiedenen Erwägungen wurden drei Klassen von Nationalstraßen vorgesehen. Die Nationalstraße erster Klasse ist vierspurig, richtungsgetrennt, kreuzungsfrei und besitzt durchgehende Abstellstreifen. Sie steht nur Motorfahrzeugen offen und ist lediglich an bestimmten Stellen zugänglich. Bei den Nationalstraßen zweiter Klasse sind nur einzelne dieser Merkmale obligatorisch wie ausschließliche Benützbarkeit durch Motorfahrzeuge und beschränkter seitlicher $\mathrm{Zu}$ tritt. Dennoch werden sie mit der ersten Klasse zu den sog. Autobahnen zusammengefaßt. Die Nationalstraßen dritter Klasse schließlich weisen die erstgenannten Kennzeichen nur in Ausnahmefällen auf. Sie stellen einfach allgemein benutzbare Überlandstraßen dar, die wenn möglich Ortschaften umfahren und höhengleiche Kreuzungen vermeiden sollen.

Die zahlreichen bautechnischen Details (Anschlüsse, Krümmungen, Profile usw.), die so gut wie alle landschaftlich einprägsam in Erscheinung treten werden, sind im Bericht selbst nachzulesen. Von der ebenfalls weiter nicht zu erwähnenden Tatsache einer eingehenden Planung der einzelnen Regionen West (Strecken Genf-Lausanne, Lausanne-Bern, Jurafuß, Lausanne-Villeneuve, Villeneuve-Sitten-Brig, Vevey -Fribourg-Bern, Vallorbe-Chavornay), Mitte (Bern-Kirchberg, Juraübergänge, Basel-Augst, Emme-Olten, Egerkingen-Hunzenschwil-Sursee, HunzenschwilZürich, Sursee-Luzern, Bern-Spiez), Innerschweiz (Zürich-Gotthard, LuzernFlüelen, Cham-Arth-Brunnen-Flüelen), Ost (Zürich-Attikon, Attikon-St. Gallen-St. Margrethen, Zürich-Pfäffikon-Ziegelbrücke, Walenstadt-Chur, Zürich -Kloten, Winterthur-Schaffhausen-Bargen, Attikon-Frauenfeld-Kreuzlingen, St. Margrethen-Sargans) und Süd (Castione-Bellinzona-Lugano-Chiasso) sowie der Alpenübergänge (Simplon, Gotthard, Bernhardin und Zufahrten) abgesehen, interessieren aber gewiß einige Gesamtdaten, von denen die Länge des Netzes von $1672 \mathrm{~km}$ gleich rund 3,3\% des gesamten Kantons- und Gemeindestraßennetzes genannt sei, wobei auf den Kanton Waadt mit $102 \mathrm{~km}$ oder 11,5\% der größte, auf Baselstadt mit $1 \mathrm{~km}$ oder $0,1 \%$ der geringste Anteil entfällt. Das neue Netz wird $90 \%$ der gesamten städtischen Bevölkerung, d. h. über 1,5 Millionen Einwohner erreichen, und innerhalb einer Zone von $0-5 \mathrm{~km}$ beidseits der Nationalstraßen werden sogar rund 3,1 Millionen Personen, 67\% der Gesamtbevölkerung, Nutzen aus ihm ziehen. Alle Städte von mehr als 40000 Einwohnern (1950) und 31 von den 42 Städten mit mehr als 10000 Einwohnern werden mit ihm verbunden. Ferner lassen sich mit Ausnahme von La Chaux-de-Fonds, Le Locle, Uster und Davos alle übrigen durch kurze Anschlußstrecken (bis $7 \mathrm{~km}$ ) verknüpfen. Nur die Kantonshauptorte Sarnen, Glarus, Appen- 
zell, Zug und Aarau werden nicht direkt vom Nationalstraßennetz bedient, doch liegen die beiden letzten ihm nahe. Außerdem schließt das Netz innerhalb der genannten 5-km-Zone 68\% der 1955 dem Fabrikgesetz unterstellten 11889 Betriebe an, wobei selbst mit beträchtlichen Erweiterungen nur kleine Verbesserungen dieses hohen Erschließungsgrades erzielt werden könnten. Dem vorgeschlagenen Straßennetz gehören die Zufahrten zu den bedeutendsten Fremdenverkehrsgebieten des Genfersees, des Wallis, des Berner Oberlandes, der Zentralschweiz, sowie des Tessins und Bündens an. Da die Nationalstraßen zudem den Anschluß an die wichtigen europäischen Durchgangsrouten sichern, tragen sie auch in dieser Hinsicht den Bedürfnissen des für die Schweiz wichtigen Tourismus Rechnung.

Insgesamt vermag also das kommende neue Straßennetz die wirtschaftlichen und Verkehrsansprüche des Landes wohl in optimaler Weise zu befriedigen. Da es die relativ hohen Kosten von 2,1 Milliarden in der ersten und 0,8 Milliarden Franken in der zweiten Bauetappe verursachen wird, ensteht die Frage, ob ein solcher Aufwand sich rechtfertigt. «Die Antwort darauf erteilt die Gegenüberstellung des Aufwandes und des von den Autobahnen geschaffenen Nutzens. Nutzen schaffend ist bereits der Einsatz produktiver Mittel für die Erstellung solcher Verkehrsanlagen, weil die direkt an den Bauarbeiten beteiligten Wirtschaftszweige eine Belebung erfahren und diese ihrerseits in die kleinsten und weitverzweigtesten Kanäle des Wirtschaftslebens weiterleiten. Diese positiven Auswirkungen auf die Volkswirtschaft lassen sich nicht ohne weiteres in Zahlen ausdrücken. Dagegen wird die volkswirtschaftliche Bedeutung der Autobahnen offensichtlicher, wenn den Jahreskosten die Ersparnisse gegenübergestellt werden, die der Motorfahrzeugverkehr beim Bestehen solcher Straßen erzielt. Sie ergeben sich aus der Verminderung der Betriebs- und Unterhaltskosten, des Zeitaufwandes und der Unfallhäufigkeit.»

Sorgfältige Berechnungen im Vergleich mit dem Ausland veranschlagen die jährliche Ersparnis auf über 200 Millionen Franken, wobei allein auf die Verminderung von Unfällen etwa 25 Millionen entfallen. Darin sind die zahlenmäßig kaum zu schätzenden volkswirtschaftlichen Auswirkungen auf die erschlossenen Gegenden natürlich nicht enthalten. Durch die Verkürzung der Fahrzeit ergibt sich für den Gütertransport der nicht auf die Eisenbahnen angewiesenen Betriebe der Industrie, des Gewerbes, des Handels und der Landwirtschaft die Möglichkeit, die Transportkosten wesentlich zu senken und auch die Transportzeiten zu reduzieren. Die Autobahnen vermögen aber auch den Tourismus zu intensivieren, weil solche Anlagen erfahrungsgemäß Neuverkehr schaffen. Dank der größern Sicherheit und des bessern Fahrkomforts steckt der Reisende seine Tagesziele weiter. Die Autobahnen entlasten aber auch das bestehende Straßennetz und beseitigen viele Unzulänglichkeiten, die den Verkehrsaufwand erhöhen und für die Straßenanwohner mehr und mehr unerträglich werden (Lärm, Staub, Erschütterungen). Die Behebung oder Verminderung der Verkehrsnot in geschlossenen Ortschaften wirkt sich nicht nur in wirtschaftlicher Hinsicht aus, sondern kann auch zur Bewahrung und Neuentfachung ideeller Werte wie Erhaltung historischer Stadt- und Landschaftsbilder beitragen. Die Nationalstraßen lassen sich also unter diesen Gesichtspunkten durchaus als Grundlagen und Impulse einer optimalen alle Interessen befriedigenden Landschaftsplanung, -gestaltung und -nutzung werten.

Indes der bloße Bau von Autobahnen schließt - wie der Bericht selbst verschiedentlich andeutet - ebenso große Risiken für die Kulturlandschaftsentwicklung wie positive Möglichkeiten in sich. Es ist mit ihm keineswegs ausgemacht, da $\beta$ er eindeutig als Anreger einer weitern gesunden Landeserschließung, einer guten künftigen Landschaftsordnung wirken wird. Zweifellos ist «der Bau der Nationalstraßen für unser Land», wie H. Gutersohn kürzlich mahnend betonte, «die große Chance, die uns in ihrem Bereich zweckmäßig gegliederte Landschaften bringen können, so wie sie die Befürworter der Landesplanung anstreben; der Bau ist aber andererseits eine neue 
große Gefahr, die uns auf dem unglücklichen Weg der Vernichtung gesunder Kulturlandschaften weiterführt, wenn nicht rechtzeitig die notwendigen landesplanerischen Maßnahmen getroffen werden». Der Landesplanung als der Treuhänderin gesunder Landschaftsentwicklung erwachsen hieraus, wie der Landschaftsgestaltung, ebenso dringliche wie dankbare Aufgaben. Es stehen ihr zur Lösung außer dem Mittel der Integralmeliorationen (Güterzusammenlegung im Zusammenhang mit Landexpropriationen, Entwässerungen und Bewässerungen, Umsiedlungen usw.) vor allem Maßnahmen der Orts- und Regionalplanung zur Verfügung.

Als grundlegende Vorarbeiten hierzu haben aber noch weitere systematische Untersuchungen über die möglichen und mutmaßlichen direkten und indirekten Auswirkungen des Straßenbaus auf die einzelnen Gegenden des Landes z. B. hinsichtlich der Verlagerungen und Vergrößerungen von Industrien und Siedlungen (insbesondere im weitern Umkreis der großen Anschlüsse), der In- und Extensivierung der Landwirtschaft, des Waldbaus, des Landschafts(Natur- und Heimat)schutzes und des Verkehrs selbst angestellt $\mathrm{zu}$ werden. Insbesondere ist in diesem Zusammenhang das Verhältnis zu den übrigen Verkehrsträgern (Bahnen, Schiffahrt, Luftfahrt) vertieft zu untersuchen, das im Bericht - wie erwähnt bewußt - unerörtert blieb. Ihre Ergebnisse können, in kommunalen und regionalen Plänen niedergelegt, $z u$ wirkungsvollen Mitteln positiver Landschaftsentwicklung in der nähern und weitern Umgebung der neuen Straßen, ja im ganzen Lande werden. Es zeigt sich hieraus, da $\beta$ mit der Planung ihres Netzes die Aufgaben ihrer Gestaltung noch gewachsen sind; dies wird auch klar, wenn berücksichtigt wird, daß der geschätzte Landbedarf von rund 3000 ha sich zu nahezu 75\% auf Mittelland- und Juragebiet, auf Zonen besten Kulturlandes richtet, das bereits in Gefahr steht, durch die unaufhaltsam fortschreitende Überbauung aufgezehrt zu werden. Die hieraus erwachsenden Probleme sind unzweifelhaft so komplex, daß sie sich nur mittelst optimaler Koordination der lokalen mit den interkommunalen, kantonalen und nationalen Behörden, Verbänden und Fachleuten sinnvoll und zu Gunsten einer positiven Zukunftsentwicklung des Volkes lösen las̈sen. Hierzu sind nicht allein die Planer und Landschaftsgestalter, sondern auch die Geographen als Erforscher der Landschaft, als Fachleute wie als Bürger aufgerufen. Der Planungsbericht gibt ihnen ein ausgezeichnetes Instrument für ihre künftigen Studien im Verkehrssektor an die Hand, das sie hoffentlich zu nutzen verstehen werden.

\section{LITERATUR}

Außer dem im Text genannten Originalbericht wurden noch benutzt: Das schweizerische Nationalstraßennetz. Zusammenfassender Bericht der Kommission des Eidg. Departements des Innern für die Planung des Hauptstraßennetzes. Bern 1958. Eidg. Drucksachen- und Materialzentrale. - RUcklI, R.: Nationalstraßenbau und Landschaftsschutz. Straße und Verkehr 46, 196o, Nr. 1 (Sonderdruck) - GUTERSOHN, H.: Betrachtungen zur eidgenössischen Straßenplanung. Die Autostraße 26, 1957, - Die Landesplanung und der Nationalstraßenbau. Plan 15, 1958, Nr. 6.

\section{LE VIE DI COMUNICAZIONE E LA RETE DELLE}

马.

\section{STRADE NAZIONALI}

Il rapporto finale della Commissione per la pianificazione delle strade nazionali del Dipartimento Federale dell'Interno - «La pianificazione delle strade nazionali svizzere» - rappresenta una tappa importante di una grande opera, che avrà indubbiamente un influsso decisivo sullo sviluppo del paesaggio antropico-culturale. La geografia, in quanto ricerca del paesaggio, non può di conseguenza rimanere indifferente: si cerca perciò di mettere in risalto i risultati essenziali dell'opera in rapporto alla pianificazione. Dati importanti sono la lunghezza complessiva dellà rete delle strade nazionali previste, che ammonta a $1672 \mathrm{~km}$, e la superficie totale di 3000 ha, che si distribuiscono in vario modo sul territorio elvetico. Si sottolinea inoltre che con la progettazione delle strade nazionali non si conclude l'opera di pianificazione, poichè appena ora si pongono i quesiti sostanziali della pianificazione nazionale, affinchè la nuova rete stradale possa servire nel modo migliore possibile al popolo svizzero. 